\title{
Optical Accelerator: Scaling Laws and Figures of Merit
}

\author{
Levi Schächter ${ }^{(1)}$, Robert L. Byer ${ }^{(2)}$ and Robert H. Siemann ${ }^{(3)}$ \\ ${ }^{(1)}$ Electrical Engineering Department, Technion - IIT, Haifa 32000, ISRAEL \\ ${ }^{(2)}$ Department of Applied Physics, Stanford University- MC4085, Stanford CA 94305, USA \\ ${ }^{(3)}$ SLAC, MS-7, Stanford University, P.O. Box 4349, Stanford, CA 94309, USA
}

\begin{abstract}
Indications that solid-state lasers will reach wall-plug to light efficiencies of $30 \%$ or more make a laser-driven vacuum-accelerator increasingly appealing. Since at the wavelength of relevant lasers, dielectrics may sustain significantly higher electric field and transmit power with reduced loss comparing to metals, the basic assumption is that laser accelerator structures will be dielectrics. For structures that have typical dimensions of a few microns, present manufacturing constraints entail planar structures that in turn, require re-evaluation of many of the scaling laws that were developed for azimuthally symmetric structures. Moreover, structures that operate at a wavelength of a few centimeters are machined today with an accuracy of microns. In future it will not be possible to maintain 4-5 orders of magnitude difference between operating wavelength and achievable tolerance. An additional difference is, that contrary to present accelerators where the number of electrons in a micro-bunch is of the order of a $10^{10}$, in an optical structure this number drops to a few thousands. Consequently, the relative impact of individual electrons may be significantly larger. Acceleration structures with higher degree of symmetry, similar to optical fibers, have also some inherent advantages however thermal gradients as well as heat dissipation may become critical impediments. The impact of all these factors on the performance of a laser accelerator structure needs to be determined. Efficiency, wakes and emittance scaling laws that have been developed recently will be presented. It will be shown that there are some inherent advantages in combining the accelerator structure and the laser cavity in one system.
\end{abstract}

\section{INTRODUCTION}

Indications that solid-state lasers will reach wall-plug to light efficiencies of $30 \%$ or more make a laser-driven vacuum-accelerator increasingly appealing. Since at the wavelength of relevant lasers, dielectrics may sustain significantly higher electric field and transmit power with reduced loss comparing to metals, the basic assumption is that laser accelerator structures will be made of dielectrics. Open, quasi-optical structures and near-field accelerators with dimensions comparable to the wavelength are both being considered. Examples of these two are: a) the LEAP[1] crossed laser beam accelerator where the interaction between the crossed laser beams and the particles is limited by slits to satisfy the Lawson-Woodward theorem[2-3], and b) the photonic band-gap (PBG) concept where a laser pulse is guided in a dielectric structure with a vacuum tunnel bored in its center [4]. Lithography, which would 
result in planar structures, and optical fiber drawing are manufacturing techniques that seem well suited for laser driven structures that have typical dimensions of a few microns.

Structures that operate at a wavelength of a few centimeters, are machined today with an accuracy of microns. In future, it will not be possible to maintain 4-5 orders of magnitude difference between operating wavelength and the achievable tolerance since this would entail engineering of a surface at the atomic level. As a result, the size of irregularities may be of the same order of magnitude as the micro-bunches, and they may generate wakes that in turn, may alter the dynamics of electrons. Fortunately, the electromagnetic properties of materials at wavelengths that are significantly smaller than $0.1 \mu \mathrm{m}$ do not differ dramatically from these of the vacuum. This fact is expected to reduce somewhat the sensitivity to manufacturing tolerances mentioned above. For all these reasons, re-evaluation of many of the scaling laws that were developed for near-field, RF-driven, azimuthally symmetric structures is necessary.

In this note the topics are organized as follows: in the next section it is shown that for a given laser power there is an optimal number of electrons which should be accelerated in order to get maximum efficiency. Since the optimal number of electrons that can be accelerated is proportional to the square root of the laser power, it is natural to determine the maximum power sustained by the structure before breakdown occurs. This is expressed in terms of the energy per unit surface (fluence) that the material can sustain - Section 3. At optical wavelengths, dielectric materials can typically sustain higher electric fields therefore, they become natural constituents of acceleration structures. In the fourth section it is shown that since dielectrics have a frequency dependent coefficient, the number of modes excited (wake field) is by orders of magnitude smaller than in the existing S-band accelerator. In the fifth section, we consider the emittance of an asymmetric acceleration structure dictated by the manufacturing constraints and in the last section some thermal considerations are discussed.

\section{EFFICIENCY}

One of the major questions that will determine the fate of any future accelerator is the efficiency since it is natural to require acceleration of the largest number of electrons at the minimal input power. In other words, maximum efficiency. Its existence may be readily conceived for a given input power, acceleration of a small number of electrons entails low efficiency. At the other extreme, increasing the number of electrons increases the beam loading, and as a result, the gradient is reduced and again the efficiency is relatively low. In between these two extremes, maximum efficiency must occur and the question is what is its value for a given input power.

Denoting the laser power injected in the structure by $P_{\text {laser }}$ and the resulting gradient at the location of the electrons by $E_{\text {acc }}$, it is possible to define the interaction impedance as 


$$
Z_{\text {int }} \equiv \frac{\left|E_{\text {acc }} \lambda\right|^{2}}{P_{\text {laser }}}
$$

which characterizes any acceleration structure; in this expression $\lambda$ is the wavelength in the vacuum. This laser pulse accelerates a bunch $(Q)$ that as it moves in an arbitrary acceleration structure generates an electromagnetic wake. Associated with this wake there is decelerating electric field component $\left(E_{\mathrm{dec}}\right)$ which by virtue of the linearity of Maxwell's equations, must be proportional to $Q$. Therefore, without loss of generality it is possible to assume that there is an effective transverse dimension denoted by $R_{\text {eff }}$, determined by the details of the structure and the bunch, such that

$$
E_{\mathrm{dec}}=\frac{Q}{4 \pi \varepsilon_{0} R_{\mathrm{eff}}^{2}} \times 2
$$

it is tacitly assumed here that the bunch is significantly smaller than the transverse dimension where the bunch propagates - see Appendix. Three generic cases have been investigated in the past and have direct relevance to the present analysis: (i) for a relativistic $(\gamma \rightarrow \infty)$ bunch moving in a vacuum tunnel of radius $R$ bored in a dielectric medium [5], it may be shown that $R_{\text {eff }}=R$. A similar result is obtained if the dielectric is of finite width or if the dielectric structure is replaced by an arbitrary dielectric periodic structure confined by a metallic surface $\left(R_{\text {eff }}=1.124 R\right)[6]$. (ii) Analysis of a similar bunch moving at a height $h$ above a dielectric half-space [7] reveals an effective length $R_{\text {eff }}=2 h$ and a similar result is expected for a dielectric layer. Moreover, when the bunch moves parallel to a dielectric cylinder [8] of radius $R$ at a distance $h$ from axis, $R_{\text {eff }}=2(h-R)$. (iii) For a wide bunch $(\Delta)$ moving at a height $h$ above a grating of arbitrary geometry, $R_{\text {eff }}=\sqrt{2 h \Delta}$. These cases are summarized in Figure 1.

A first figure of merit may be developed by comparing the impedance linked to the acceleration mode and that seen by the wake. For this purpose it is important to bear in mind that contrary to $E_{\text {acc }}$, which is a very narrow-band signal, the spectrum of $E_{\mathrm{dec}}$ may be very broad. However, since it is possible to establish the power associated with this field, $P_{\text {wake }}=Q v E_{\text {dec }}$, then the impedance definition as in Eq.(2.1) entails

$$
Z_{\text {wake }} \equiv \frac{\left|E_{\text {dec }} \lambda\right|^{2}}{P_{\text {wake }}}=Z_{0} \frac{1}{2 \pi\left(R_{\text {eff }} / \lambda\right)^{2}}
$$




\begin{tabular}{|l|l|}
\hline Configuration & \multicolumn{1}{c|}{$R_{\text {eff }}$} \\
\hline & $R_{\text {eff }}=R$ \\
\hline & $R_{\text {eff }}=2 h$ \\
\hline & $R_{\text {eff }}=2(h-R)$ \\
\hline
\end{tabular}

FIGURE 1. The effective radius ( $\left.R_{\text {eff }}\right)$ for different configurations. 


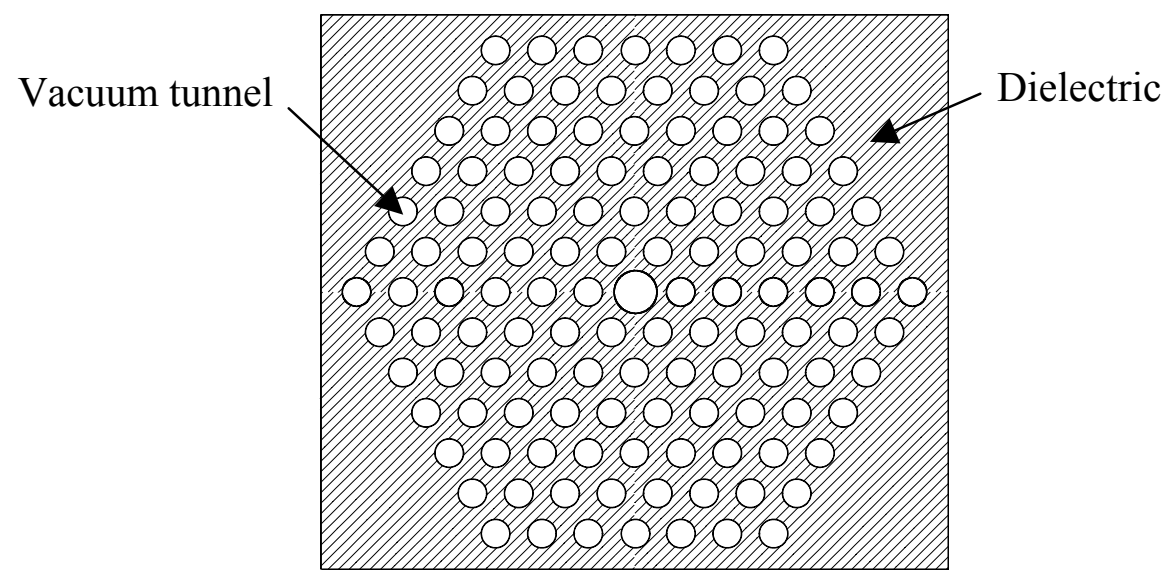

FIGURE 2. Schematic of the honey-comb photonic band gap structure analyzed in Ref. 4.

wherein $Z_{0}=\sqrt{\mu_{0} / \varepsilon_{0}}$ is the vacuum impedance. For example, the interaction impedance of the photonic band-gap structure analyzed in Ref. 4, see Figure 2, has an interaction impedance of $Z_{\text {int }}=19.5 \Omega$ at $1 \mu \mathrm{m}$ and for an accelerating gradient of $1 \mathrm{GV} / \mathrm{m}$ the total power required is about $50 \mathrm{~kW}$. Furthermore, since the radius of the vacuum tunnel is $R=0.678 \lambda$ and it was demonstrated [Ref. 9] that $R_{\text {eff }} \simeq 1.23 R$, then $Z_{\text {wake }} \simeq 86 \Omega$.

With these two impedances, it is possible to determine the effective (loaded) gradient as

$$
E_{\text {eff }} \equiv E_{\text {acc }}-E_{\text {dec }}=\frac{1}{\lambda} \sqrt{P_{\text {laser }} Z_{\text {int }}}-\frac{Q c Z_{\text {wake }}}{\lambda^{2}}
$$

therefore, the gain in the kinetic energy of the bunch in structure of length $L$ is $\Delta U_{\text {kin }} \equiv Q E_{\text {eff }} L$ whereas the total electromagnetic energy stored in the structure is $U_{\text {em }} \equiv P_{\text {laser }} \tau_{\mathrm{f}}=P_{\text {laser }} \frac{L}{c} \frac{1-\beta_{\mathrm{gr}}}{\beta_{\mathrm{gr}}}$ where $\tau_{\mathrm{f}}$ is the fill-up time and $c \beta_{\mathrm{gr}}$ is the group velocity. The expression for the fill-up time tacitly assumes full overlap of the electron bunch during the entire transit time of the electrons. Moreover, it is assumed that the laser pulse duration is shorter than the time it takes the pulse to traverse the structure, in fact, the difference between the two is taken to be equal to the bunch transit time $\tau_{\mathrm{tr}} \cong \frac{L}{c}=\frac{L}{c \beta_{\mathrm{gr}}}-\tau_{\mathrm{p}}$ implying that the minimal pulse duration is $\tau_{\mathrm{p}} \simeq \tau_{\mathrm{f}}=\frac{L}{c} \frac{1-\beta_{\mathrm{gr}}}{\beta_{\mathrm{gr}}}$. Based on these two energy definitions, the efficiency of the acceleration process is 


$$
\eta \equiv \frac{\Delta U_{\mathrm{kin}}}{U_{\mathrm{em}}}=\frac{Q\left(Q_{0}-Q\right)}{Q_{\mathrm{n}}^{2}}
$$

wherein $Q_{\mathrm{n}} \equiv \sqrt{\frac{P_{\text {laser }}}{Z_{\text {wake }}} \frac{\lambda^{2}}{c^{2}} \frac{1-\beta_{\mathrm{gr}}}{\beta_{\mathrm{gr}}}}$ and $Q_{0} \equiv \frac{\lambda \sqrt{P_{\text {laser }} Z_{\text {int }}}}{c Z_{\text {wake }}}$ is the charge for which the effective gradient vanishes - see Eq. (4). In other words, all the energy gained by the bunch from the laser field is lost to Cerenkov radiation. Clearly, maximum efficiency occurs for an optimal value of the charge given by

$$
Q_{\mathrm{opt}} \equiv \frac{1}{2} Q_{0}=\frac{\lambda \sqrt{P_{\text {laser }} Z_{\text {int }}}}{2 c Z_{\text {wake }}}
$$

in which case the value of this efficiency is

$$
\eta_{\max }=\left(\frac{1}{2} \frac{Q_{0}}{Q_{\mathrm{n}}}\right)^{2}=\frac{1}{4} \frac{\beta_{\mathrm{gr}}}{1-\beta_{\mathrm{gr}}} \frac{Z_{\text {int }}}{Z_{\text {wake }}}
$$

implying that the maximum efficiency is determined by the ratio of the impedances and the group velocity. For the PBG structure mentioned above [4], the group velocity is $0.58 \mathrm{c}$ therefore the maximum possible efficiency is $7.86 \%$. It is likely that considerations of energy spread will lead to charges and efficiencies below the optimum values.

\section{BREAK-DOWN LIMIT}

The optimal number of electrons to be accelerated is determined by the laser intensity and the latter is limited primarily by the surface threshold limit expressed in terms of energy impinging per unit surface i.e. the threshold damage fluence $F$. Based on Ref. 10 there is a relatively simple model for this quantity namely,

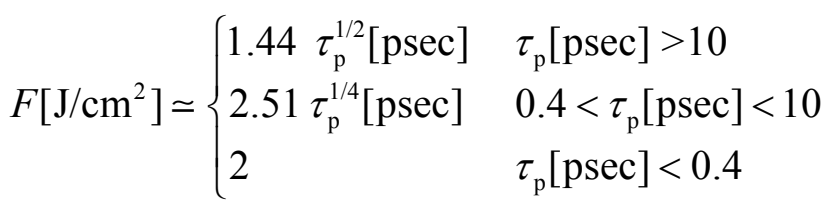

However, while it was relatively simple to determine the amount of energy in a structure based on the power and the fill-up time, it is difficult to provide a general relation between the maximum field $\left(E_{\max }\right)$ and the accelerating gradient $E_{\text {acc }}$. Nevertheless, by virtue of the linearity of Maxwell's equations, similar to Eq.(1), it is possible to determine an impedance 


$$
Z_{\text {max }} \equiv \frac{\left|E_{\max } \lambda\right|^{2}}{P_{\text {laser }}}
$$

which is a characteristic of the structure. Based on this definition and on Eq.(1) it is evident that

$$
\frac{Z_{\max }}{Z_{\text {int }}}=\frac{E_{\max }^{2}}{E_{\text {acc }}^{2}} .
$$

Furthermore, by calculating the maximum field for a given gradient and knowing the interaction impedance in the structure, the impedance $Z_{\max }$, is set. One step further is achieved by realizing that the maximum amount of energy impinging upon a unit surface (fluence) of the structure is roughly determined by the energy density in the region of the maximal field, the group velocity and the pulse duration or explicitly,

$$
F=\left(\frac{1}{2} \varepsilon_{0} \varepsilon E_{\max }^{2}\right) c \beta_{\mathrm{gr}} \tau_{\mathrm{p}} .
$$

Note that, for example, the radius of curvature i.e. the smallest geometric parameter of the structure is tacitly considered to determine the maximum field $E_{\max }$. Consequently, based on Eqs. (8-9) and using Eq.(11) the maximum laser power is given by

$$
P_{\text {laser }}^{(\max )}=\frac{2 \lambda^{2} F}{\varepsilon \beta_{\mathrm{gr}} \tau_{\mathrm{p}}} \frac{Z_{0}}{Z_{\mathrm{int}}}\left(\frac{E_{\mathrm{acc}}}{E_{\max }}\right)^{2} .
$$

Accordingly, based on Eq.(6)

$$
Q_{\mathrm{opt}}^{(\max )}=\frac{\lambda^{2}}{\sqrt{\varepsilon}} \frac{Z_{0}}{Z_{\text {wake }}} \frac{E_{\mathrm{acc}}}{E_{\max }} \sqrt{\frac{\varepsilon_{0} F}{2 \beta_{\mathrm{gr}} c \tau_{\mathrm{p}}}} .
$$

For the PBG structure it was found that $E_{\max }=2.1 E_{\mathrm{acc}}$. The main relations so far are summarized in the next table. 
TABLE 1. The parameters of the photonic band gap structure are: rod spacing $a / \lambda=1.3$, radius of the central cylinder $R / \lambda=0.678$, the interaction impedance $Z_{\text {int }}=19.5 \Omega$, group velocity $\beta_{\mathrm{gr}}=0.58$, the effective radius $R_{\text {eff }}=1.23 R$ and the ratio of the accelerating and maximum surface field $E_{\max } / E_{\text {acc }}=2.1$.

\begin{tabular}{|l|l|l|}
\hline \multicolumn{1}{|c|}{ Quantity } & \multicolumn{1}{|c|}{ General Expression } & \multicolumn{1}{c|}{$\begin{array}{c}\text { PBG (Ref. 4) } \\
\tau_{\mathrm{p}}=200 \mathrm{psec}\end{array}$} \\
\hline Number of electrons & $Q_{\mathrm{opt}}^{(\max )}=\frac{\lambda^{2}}{\sqrt{\varepsilon}} \frac{Z_{0}}{Z_{\text {wake }}} \frac{E_{\mathrm{acc}}}{E_{\mathrm{max}}} \sqrt{\frac{\varepsilon_{0} F}{2 \beta_{\mathrm{gr}} c \tau_{\mathrm{p}}}}$ & $N_{\mathrm{el}}=6.5 \times 10^{4}$ \\
\hline Laser power & $P_{\text {laser }}^{(\max )}=\frac{2 \lambda^{2} F}{\varepsilon \beta_{\mathrm{gr}} \tau_{\mathrm{p}}} \frac{Z_{0}}{Z_{\mathrm{int}}}\left(\frac{E_{\mathrm{acc}}}{E_{\mathrm{max}}}\right)^{2}$ & $P_{\text {laser }}^{(\max )}=15.8[\mathrm{~kW}]$ \\
\hline Maximum gradient & $E_{\mathrm{acc}}^{(\max )}=\frac{1}{\lambda} \sqrt{P_{\mathrm{laser}}^{(\max } Z_{\mathrm{int}}}$ & $E_{\mathrm{acc}}^{(\max )}=0.55[\mathrm{GV} / \mathrm{m}]$ \\
\hline Decelerating field & $E_{\mathrm{dec}}=\frac{Q}{4 \pi \varepsilon_{0} R_{\mathrm{eff}}^{2}} \times 2$ & $E_{\mathrm{dec}}=0.27[\mathrm{GV} / \mathrm{m}]$ \\
\hline Maximum efficiency & $\eta_{\mathrm{max}}=\frac{1}{4} \frac{\beta_{\mathrm{gr}}}{1-\beta_{\mathrm{gr}}} \frac{Z_{\text {int }}}{Z_{\text {wake }}}$ & $\eta_{\mathrm{max}}=7.86 \%$ \\
\hline
\end{tabular}

\section{FREQUENCY DEPENDENCE OF $\varepsilon$}

One of the major differences between a future (optical) accelerator based on a dielectric material and the current systems based on metallic structures is the fact that the latter supports excitation and propagation of many thousands of modes other than the main accelerating mode whereas the former, may support only a few modes before the material becomes transparent. In order to quantify this statement we shall consider the simplest model possible: a point-charge moving at a constant velocity $(v)$ in an azimuthally symmetric waveguide filled with a dielectric material $(\varepsilon)$. Propagating modes develop provided the poles are real quantities namely, the zeros of the function

$$
\omega^{2}+\frac{p_{s}^{2} c^{2}}{R^{2}} \frac{\beta^{2}}{1-\varepsilon(\omega) \beta^{2}}=0
$$

are real; here $R$ is the radius of the waveguide and $p_{s}$ represents the zeros of the zeroorder Bessel function of the first kind $s=1,2, \ldots$. Assuming that

$$
\varepsilon(\omega)=\left\{\begin{array}{cc}
\varepsilon_{\mathrm{r}} & \omega<\omega_{\mathrm{c}} \\
1 & \omega>\omega_{\mathrm{c}}
\end{array}\right.
$$


then for a relativistic particle the propagating modes are limited to $\mathrm{s}_{\mathrm{c}} \mathrm{s}_{\mathrm{c}}$ where

$$
s_{c} \approx \operatorname{Integer}\left(\frac{\omega_{c}}{c} R \sqrt{\varepsilon_{r}-1} \frac{1}{\pi}\right) .
$$

Higher modes do not contribute to radiation and as such, all the electromagnetic (evanescent) field corresponding to these higher modes is attached to the bunch. In order to better quantify this statement let us consider two examples; in both cases the "cut-off" of the material is at $\lambda_{\mathrm{c}}=200 \mathrm{~nm}$ and $\varepsilon_{\mathrm{r}}=2$. In an S-band structure the typical radius is of the order of centimeters $(\mathrm{R}=1 \mathrm{~cm})$ therefore $\mathrm{s}_{\mathrm{c}}=10^{5}$ whereas for an optical system the typical radius is $\mathrm{R}=1 \mu \mathrm{m}$ thus $\mathrm{s}_{\mathrm{c}}=10$ !! Clearly, expression (16) constitutes a quantitative measure for the number of modes in a typical structure whose transverse dimension is $R$ and $\omega_{\mathrm{c}}$ is the "cut-off" angular frequency of the dielectric material.

\section{EMITTANCE}

One of the important obstacles in the process of implementing an optical vacuum accelerator is the constraint imposed by the emittance therefore, a few comments regarding the scaling laws linked to the emittance in a non-azimuthally symmetric structure are in place. In order to have the correct orders of magnitude, one should bear in mind that in an azimuthally symmetric structure, the ratio of the transverse force to the longitudinal force is virtually negligible since it is proportional to

$$
\left|\frac{F_{\perp}}{F_{z}}\right| \approx \frac{1}{4 \gamma^{2}}\left(\frac{\omega}{c} R_{b}\right) ;
$$

for example, typical values are $\gamma \sim 10^{4}, \omega R_{b} / c \sim 1$ wherein $R_{\mathrm{b}}$ is the radius of the bunch. On the other hand, in a non-symmetric structure of a typical transverse dimension $a$, see Figure 3 for a typical configuration, the ratio of the two forces is

$$
\left|\frac{F_{\perp}}{F_{z}}\right| \approx\left(\frac{\omega}{c} a\right)^{-1}
$$

$3 \mathrm{D}$ numerical simulations of a bunch of $30 \mathrm{GeV}$ electrons indicate that the relative emittance change is drastically affected by the transverse dimension

$$
\frac{\varepsilon^{(\mathrm{out})}-\varepsilon^{(\mathrm{in})}}{\varepsilon^{(\mathrm{in})}} \simeq\left(\frac{b}{a}\right)^{v}
$$


wherein it was assumed that the bunch experiences a gradient of $1 \mathrm{GV} / \mathrm{m}$ along a $10 \mathrm{~cm}$ long structure and the initial bunch length was $90^{\circ}$ of optical phase. The parameters $b$ and $v$ depend on the radius of the beam and the corresponding emittance:

$$
\begin{array}{lll}
x: & b=20-1.54 R_{b} & v=2.4+1.15 R_{b}-0.16 R_{b}^{2} \\
y: & b=10.5-R_{b} & v=1.1+1.85 R_{b}-0.23 R_{b}^{2}
\end{array}
$$

therefore in case $R_{b}=3 \mu \mathrm{m}$ the coefficients for $\varepsilon_{\mathrm{x}}$ are $b_{\mathrm{x}}=15.4$ and $\nu_{\mathrm{x}}=4.4 ; a, b$ and $R_{\mathrm{b}}$ are expressed in microns. . Bunch length $(\Delta \chi)$ also affects the emittance. A similar analysis shows that if $|\Delta \chi|<90^{\circ}$, the emittance change is quadratic in $\Delta \chi$

$$
\frac{\varepsilon^{(\mathrm{out})}-\varepsilon^{(\mathrm{in})}}{\varepsilon^{(\mathrm{in})}}=1.1 \times\left(\frac{\Delta \chi}{180^{\circ}}\right)^{2} .
$$

here $R_{\mathrm{b}}=3 \mu \mathrm{m}$ and $a=20 \mu \mathrm{m}$. According to the emittance growth in a single stage, two drawbacks are evident: in order to keep the emittance growth moderate it is necessary to have a relatively large transverse dimension (typically a few vacuum wavelengths) implying operation in a multi-mode regime. And even then, according to simulations, the increase in the emittance is almost $10 \%$ in a $10 \mathrm{~cm}$ long segment. In order to overcome this impediment due to the asymmetry of the structure it is quite natural to "symmetrized" the structure. This is done by constructing each module by sets of four non-symmetric segments each one rotated by $90^{\circ}$. In this way, the average value of the transverse field components is zero. However, there remain two questions to be addressed: (i) is the zero average transverse field condition sufficient to maintain the low emittance over extended length? (ii) What would be a reasonable division in a typical acceleration module?

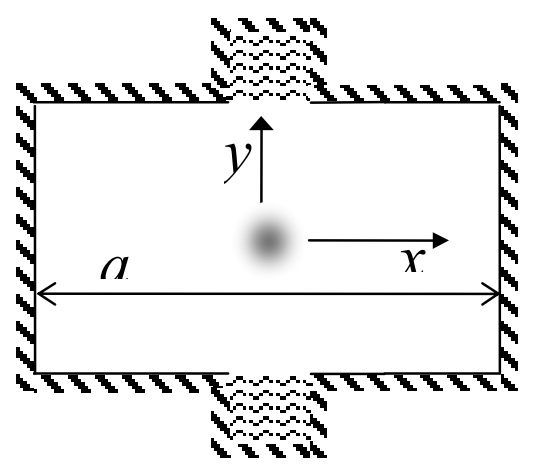

FIGURE 3.: Schematic of one possible configuration of a non-symmetric acceleration structure. Emittance variation along such a segment is strongly dependent on $a$. 
In order to address these questions a one meter long module was simulated. The two plates are $a=20 \mu \mathrm{m}$ apart, the initial radius of the bunch is $R_{\mathrm{b}}=2 \mu \mathrm{m}$, its average energy is $30 \mathrm{GeV}$ with an energy spread of $1 \%$, its length being $3.6^{\circ}$ on the scale of a $1.06 \mu \mathrm{m}$ laser wavelength which in turn generates at the electrons location a gradient of $1 \mathrm{GV} / \mathrm{m}$. For these parameters, the emittance at the output approaches the limit of a symmetric structure when using a large number of modules, specifically,

$$
\frac{\varepsilon^{(\mathrm{out})}-\varepsilon^{(\mathrm{in})}}{\varepsilon^{\text {(in) }}} \simeq \frac{4.14}{1+\left(\frac{N_{s t}}{15}\right)^{2}}
$$

where $N_{\text {st }}$ is the number of segments. For 400 segments the emittance increase in a $1 \mathrm{~m}$ long acceleration structure is $0.6 \%$, entailing a maximum segment length of $2.5 \mathrm{~mm}$.

\section{THERMAL CONSIDERATIONS}

Although dielectric material are known to have low ohm loss there still is an important aspect of temperature gradients and heat dissipation. Electromagnetic field variations in the cross section of the structure are inevitable and consequently, so are temperature gradients since electromagnetic power is partially absorbed by the dielectrics. Reduction of these gradients is possible if: (i) the material used has good thermal conductivity and (ii) heat dissipation at the outer boundary is properly designed. In order to have some order of magnitude, consider a $25 \mathrm{~cm}$ long module of an outer circumference of $40 \mu \mathrm{m}$ in which we inject $15 \mathrm{~kW}$ of laser power, the loss of the material is $\tan \delta=10^{-4}$. For this particular set of parameters the total amount of power that needs to be dissipated is of the order of $15 \mathrm{~W} / \mathrm{cm}^{2}$ which is two orders of magnitude lower that what we may anticipate to be able to dissipate $I_{D} \sim 1.5 \mathrm{~kW} / \mathrm{cm}^{2}$. However, special attention is required here since heat dissipation engineering, at the micron-scale favors planar configuration.

\section{DISCUSSION}

The fate of any future linear collider will be determined by the energy efficiency of the entire system. Typical collider designs $(500 \mathrm{GeV})$ predict $8 \mathrm{MW}$ of average beam power assuming $10^{14}$ electrons/sec. At an acceleration efficiency of $7.86 \%$ as calculated in Section 2 for the PBG, this entails $0.1 \mathrm{GW}$ of average laser power and assuming a wall-plug to light efficiency of $30 \%$, the total amount of average power is about $0.34 \mathrm{GW}$. For accelerating $10^{14}$ electrons/sec the bunch repetition rate of the system would need to be about $1 \mathrm{GHz}$ since the optimal number of electrons per bunch was demonstrated to be $10^{5}$. 
All laser based schemes rely on the fact that a relatively small fraction of the energy stored in the laser cavity is extracted and used in the acceleration structure. Conceptually, it seems possible to take advantage of the high intensity electromagnetic field that develops in the cavity and incorporate the acceleration structure in the laser cavity as illustrated in Figure 4.

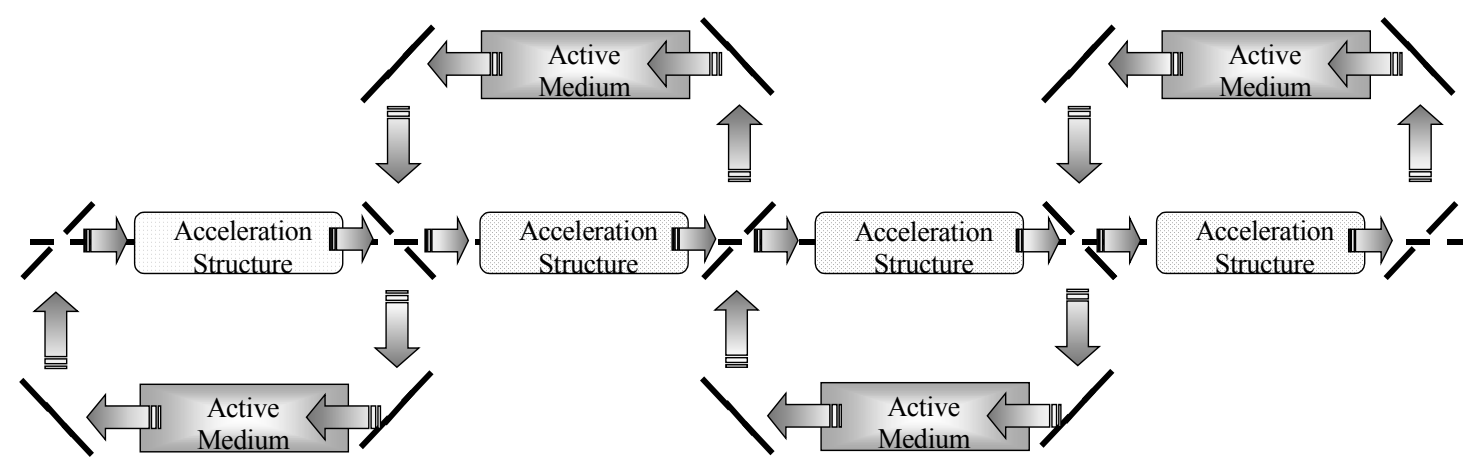

FIGURE 4.: Schematic of 4 segments of combined acceleration structure and laser cavity.

By choosing the round-trip time of the laser cavity to be equal to the repetition rate of the system, it is possible to use the wake generated by the macro-bunch $v$, amplify it in the active medium and further inject it in the acceleration structure for accelerating the macro-bunch $v+1$. Nominally, this may lead to a significantly higher overall efficiency since energy is "re-cycled".

\section{ACKNOWLEDMENTS}

This study was supported by the United States Department of Energy and the Israel Science Foundation.

\section{REFERNCES}

1. Huang Y. C., Zheng D., Tulloch WM. and Byer R.L., Appl. Phys. Lett. 68, 753-5 (1996).

2. Lawson J. D., IEEE Trans. Nucl. Sci. NS-26, 4217-9 (1979).

3. Woodward P. M., J. IEE 93, 1554 (1947)

4. Xintian Eddie Lin, Phys. Review Special Topics - Accelerators and Beams, 4, 051301 1-7 (2001).

5. Schächter L. and Schieber D., NIM A, 388, 8-16 (1997).

6. Schächter L. and Schieber D., Physics Letters A., 293, 17-22 (2002).

7. Schieber D. and Schächter L., Physical Review E., 57, 6008-6015 (1998).

8. Schieber D. and L. Schächter L., Physical Review E, 64, 056503 1-8 (2001).

9. Schächter L. Wake-Field in a Photonic Band Gap Structure, Private communication

10. Stuart B.C., Feit M.D., Rubenchick A.M., Shore B.W. and Perry M.D.,Phys. Rev. Lett., 74(12), 2248-2251 (1995). 


\section{APPENDIX}

Based on the analysis in Ref. 5 the longitudinal electric field acting on a relativistic point-charge $(Q)$ as it moves along the axis of a vacuum channel of radius $\mathrm{R}$ bored in a dielectric medium $(\varepsilon)$ is given by

$$
E_{z}(\tau=t-z / v)=\frac{Q}{4 \pi \varepsilon_{0} R^{2}} \frac{1}{\pi} \int_{-\infty}^{\infty} d x \frac{\xi}{I_{0}^{2}(|x|)+\xi^{2} I_{1}^{2}(|x|)} e^{j x \gamma \beta c \tau / R}
$$

wherein $\xi \simeq \gamma \sqrt{\varepsilon-1} / \varepsilon$; note that the radial dependence is omitted here since it was found that for a relativistic bunch $(\gamma \gg 1)$ the transverse dimension has a negligible effect. In case of a bunch of duration $\tau_{\mathrm{p}}$, the average electric field that acts on the bunch is

$$
\langle E\rangle=\frac{Q}{4 \pi \varepsilon_{0} R^{2}} \frac{1}{\tau_{p}} \int_{-\tau_{p} / 2}^{\tau_{p} / 2} d \tau \frac{1}{\tau_{p}} \int_{-\tau_{p} / 2}^{\tau_{p} / 2} d \tau^{\prime} \frac{1}{\pi} \int_{-\infty}^{\infty} d x \frac{\xi}{I_{0}^{2}(|x|)+\xi^{2} I_{1}^{2}(|x|)} e^{j x \gamma \beta c\left(\tau-\tau^{\prime}\right) / R}
$$

At the limit of $\gamma \rightarrow \infty$ the contribution to the first integral is from the region $x \ll 1$ since $\xi \propto \gamma$ therefore

$$
\langle E\rangle=\frac{Q}{4 \pi \varepsilon_{0} R^{2}} 4 \frac{1}{\tau_{p}} \int_{-\tau_{p} / 2}^{\tau_{p} / 2} d \tau \frac{1}{\tau_{p}} \int_{-\tau_{p} / 2}^{\tau_{p} / 2} d \tau^{\prime} \frac{1}{2 \pi} \int_{-\infty}^{\infty} d u \frac{e^{j u 2 \gamma c\left(\tau-\tau^{\prime}\right) / \xi R}}{1+u^{2}} .
$$

The last integral can be evaluated analytically

$$
\frac{1}{2 \pi} \int_{-\infty}^{\infty} d u \frac{e^{j u \chi}}{1+u^{2}}=\frac{1}{2} e^{-|\chi|}
$$

and it is convenient to define $\bar{\tau}_{p} \equiv \tau_{p} \gamma c / R \xi$ enabling to calculate analytically the other two integrals

$$
\langle E\rangle=\frac{Q}{4 \pi \varepsilon_{0} R^{2}} \times \frac{2}{\bar{\tau}_{p}}\left(1-e^{-\bar{\tau}_{p}} \frac{\sinh \left(\bar{\tau}_{p}\right)}{\bar{\tau}_{p}}\right) .
$$

Two comments are in place at this stage: firstly, the average decelerating field is independent of $\gamma$ since $\bar{\tau}_{p}=c \tau_{p} \varepsilon / R \sqrt{\varepsilon-1}$. Secondly, within an excellent approximation this relation may be expressed by 


$$
\langle E\rangle \simeq \frac{Q}{4 \pi \varepsilon_{0} R^{2}} \times \frac{2}{1+\bar{\tau}_{p}} .
$$

If the pulse duration is significantly shorter than the transverse dimension of the channel, $\bar{\tau}_{p} \ll 1$,

$$
\langle E\rangle \simeq \frac{Q}{4 \pi \varepsilon_{0} R^{2}} \times 2
$$

whereas at the other extreme $\left(\bar{\tau}_{p} \gg 1\right)$,

$$
\langle E\rangle \simeq \frac{Q}{4 \pi \varepsilon_{0} R^{2}} \times \frac{R}{c \tau_{p}} \frac{2 \varepsilon}{\sqrt{\varepsilon-1}} .
$$

